\title{
Performance of Chilli (Capsicum annuum L.) Genotypes for Yield and Yield Attributing Traits
}

\author{
Mopidevi M Nagaraju ${ }^{1} *$, R.V.S.K. Reddy ${ }^{2}$, K. Madhavi Reddy ${ }^{3}$, L. Naram Naidu ${ }^{4}$, \\ A. Snehalata $\operatorname{Rani}^{5}$ and K. Uma Krishna ${ }^{6}$
}

${ }^{1}$ Department of Vegetable Science, ${ }^{6}$ Department of Statistics, College of Horticulture, Dr. Y.S.R Horticultural University, Venkataramannagudem - 534 101, Andhra Pradesh, India ${ }^{2}$ Director of Extension, Dr. Y.S.R Horticultural University, Venkataramannagudem - 534 101, Andhra Pradesh, India

${ }^{3}$ Division of Vegetable Crops, ICAR - Indian Institute of Horticultural Research (IIHR), Bengaluru, Karnataka, India

${ }^{4}$ Horticultural Research Station, Dr. Y.S.R Horticultural University, Lam Guntur, Andhra Pradesh, India

${ }^{5}$ Horticultural Research Station, Kovvur, West Godavari District, Andhra Pradesh, India

*Corresponding author

\section{Keywords}

Capsicum annuum L., Chilli,

Genotypes, Growth, Yield

Article Info

\section{Accepted:}

17 November 2018

Available Online:

10 December 2018
A B S T R A C T

An experiment was conducted during kharif 2017-2018 at College of Horticulture, Venkataramannagudem, Dr. YSR Horticultural University, Andhra Pradesh (India) to identify potential genotypes for twelve quantitative traits among fifty three genotypes of chilli (Capsicum annuum L.). The analysis of variance revealed significant differences among the genotypes for all the twelve characters indicating the presence of genetic variability among the genotypes. Among fifty three genotypes, the genotype Meghalaya Local recorded maximum plant height, plant spread and number of primary branches per plant. Whereas, the genotype IHR 2900 recorded the earliest for days to 50 per cent flowering and days to 50 per cent ripening. However, the maximum red ripe fruit yield was recorded in genotype IHR 4611. Meghalaya Local recorded maximum number of fruits per plant, whereas, California Wonder was recorded maximum fruit length, fruit width and fruit weight. The genotype IHR 3478 recorded highest number of seeds per fruit, whereas, the genotype IHR 4604 recorded highest 1000 seed weight. The characters showing wide range of variation provide an ample scope for selecting superior types and the selected genotypes can be used in further breeding programme.

\section{Introduction}

Chilli (Capsicum annuum L.) an important spice cum vegetable crop, is extensively cultivated in India both under rainfed and irrigated conditions. Chilli belongs to the genus Capsicum of family Solanaceae. It has originated in Mexico, Southern Peru and 
Bolivia. There are mainly five cultivated Capsicum spp. viz., C. annuum, C. baccatum, $C$. chinense, $C$. frutescens, and $C$. pubescens of which Capsicum annuum is the dominant species world over and could be broadly classified into non-pungent (sweet pepper) and pungent (chilli or hot pepper) based on their level of pungency (Bosland and Votava, 2000). In India, it is an important ingredient in daily cuisine and is a rich source of vitamin A, $\mathrm{E}, \mathrm{C}$ and potassium. It is also a good source of oleoresin which has good export potential.

India is the largest producer, consumer and exporter of chilli with an annual production of 2.09 million tonnes from 0.84 million ha (National Horticulture Board, 2016-17). According to an estimate for 2016, in India, chillies (dry-red and fresh-green fruits) were cultivated in 797,029 ha with a total production of 1.3 million tonnes of dry fruits and 679,17 tonnes of fresh fruits. Average yield of dry chilli harvest was around $1.7 \mathrm{t} / \mathrm{ha}$ compared to that of $8.4 \mathrm{t} / \mathrm{ha}$ for green chilli (FAOSTAT, 2016). In India, the states of Andhra Pradesh, Telangana, Karnataka, Maharashtra, Orissa and Tamil Nadu account for more than $75 \%$ of the area and production of chilli.

The alkaloid 'capsaicin' present in placenta of the chilli fruit responsible for its pungency has diverse prophylactic and therapeutic uses in Allopathic and Ayurvedic medicine (Sumathy and Mathew, 1984) and directly scavenge various free radicals (Kogure et al., and 2002; Bhattacharya et al., 2010) and has wide applications in the food, medicine and pharmaceutical industries. Chilli is a good source of vitamin $\mathrm{C}$ (ascorbic acid) used in food and beverage industries (Bosland and Votava, 2000). It has also acquireda great importance because of the presence of 'oleoresin', which permits better distribution of color and flavor in foods. The assessment of nature and magnitude of variability in the available germplasm is the prerequisite of any breeding programme. The effectiveness of selection and development of improved varieties depends on the nature of variability expressed for yield and its contributing characters in the gene pool. High yield and yield contributing characters with improved quality parameters have been the major objective of chilli breeding programme. The importance of genetically diverse genotypes with desirable combinations has also been realized by several workers (Peter and Rai, 1978 and Das et al., 1998). Keeping in view the above facts, the present investigation was undertaken to observe the performance of genotypes of chilli for quantitative traits and to screen the best performing genotypes for utilization in further breeding programme.

\section{Materials and Methods}

The experiment was carried out with 53 chilli genotypes (Table 1) at Collage of Horticulture, Venkataramannagudem, Dr. YSR Horticultural University, Andhra Pradesh, India during 2017-2018 in a randomized block design with three replications. Each genotype was raised in 3.6 $\mathrm{m} \times 1.8 \mathrm{~m}$ plot size with a spacing of $60 \times 45$ $\mathrm{cm}$ accommodating 24 plants per plot. The crop was grown with standard package of practices. Five competitive plants were selected at random for recording the observations on 12 characters viz., plant height $(\mathrm{cm})$, plant spread $\left(\mathrm{cm}^{2}\right)$, number of primary branches per plant, days to 50 per cent flowering, days to 50 per cent ripening, number of fruits per plant, fruit length $(\mathrm{cm})$, fruit width $(\mathrm{cm})$, fruit weight $(\mathrm{cm})$, number of seeds per fruit, seed weight (g/1000 seed) and red ripe fruit yield per plant $(\mathrm{g})$. The crop was raised as per the recommended package of practices. Analysis of variance was carried out as per the procedure given by Panse and Sukhatme (1985). 


\section{Results and Discussion}

The analysis of variance (Table 2) revealed significant differences among the genotypes for all the twelve characters studied indicating the presence of genetic variability in the genotypes and considerable scope for their improvement. These results are in conformity with earlier reports of Farhad et al., (2008), Gupta et al., (2009), Suryakumari et al., (2010) and Kumar et al., (2012) in chilli.

Plant height of genotypes varied from 39.40 to $109.33 \mathrm{~cm}$ with a mean of $65.60 \mathrm{~cm}$ (Table 3 ). Among the genotypes, Meghalaya Local $(109.33 \mathrm{~cm})$ recorded maximum plant height followed by IHR $4597(88.40 \mathrm{~cm})$, whereas, minimum plant height was recorded in IHR $3024(39.40 \mathrm{~cm})$. Twenty five genotypes recorded significantly more plant height than grand mean. The trait, plant spread varied from 47.05 to $108.07 \mathrm{~cm}^{2}$ with over all mean of $62.79 \mathrm{~cm}^{2}$. Significantly highest plant spread was observed in Meghalaya Local (108.07 $\mathrm{cm}^{2}$ ) followed by BhutJolokia $\left(92.07 \mathrm{~cm}^{2}\right)$, whereas, IHR $1485\left(47.05 \mathrm{~cm}^{2}\right)$ recorded lowest plant spread. Plant spread of twenty four genotypes was significantly higher than grand mean. The mean values for number of branches per plant varied from 2.20 to 5.33 with a grand mean of 3.44. Number of primary branches per plant was least in IHR 3449 (2.20), significantly highest number of primary branches per plant was recorded in Meghalaya Local (5.33). Twenty six genotypes recorded significantly higher number of primary branches than grand mean. These results are in line with findings of Munshi et al., (2010) and Nehru et al., (2012), who also reported highest variability for above traits. The mean values for number of days to 50 per cent flowering ranged from 27.33 to 52.67 days with a general mean of 37.64 days. The genotype IHR 2900 was found to be early (27.33 days) followed by IHR 3315 (29.67 days), whereas, Bhut Jolokia (52.67days) recorded maximum number of days for 50 per cent flowering. Twenty five genotypes recorded less number of days to 50 per cent flowering compared to grand mean. The mean values for number of days to 50 per cent ripening ranged from 68.67 to 97.67 days with a general mean of 81.07 days. The genotype IHR 2900 was found to be early (68.67 days), closely followed by IHR 3315 (69.00 days), whereas Meghalaya Local (97.67 days) recorded maximum number of days to 50 per cent ripening. Twenty three genotypes recorded less number of days to 50 per cent ripening compared to grand mean. Bharadwaj et al., (2007), Tembhurne et al., (2008) and Arup et al., (2011) reported same trends of flowering in chilli.

The number of fruits per plant varied from 11.44 to 798.67 with an overall mean of 197.09. Significantly highest number of fruits per plant was observed in Meghalaya Local (798.67) followed by IHR 4597 (384.11), whereas, California Wonder (11.44) recorded the least number of fruits. Thirty one genotypes recorded significantly more number of fruits compared to grand mean. Fruit length varied from 2.15 to $14.48 \mathrm{~cm}$ with an overall mean of $8.12 \mathrm{~cm}$. Highest fruit length was recorded in California Wonder $(14.48 \mathrm{~cm})$ which, was on par with IHR $4611(14.24 \mathrm{~cm})$, whereas, the lowest fruit length was recorded in Meghalaya Local $(2.15 \mathrm{~cm})$. Twenty five genotypes recorded significantly more fruit length than overall mean. The character, fruit width ranged from 2.09 to $22.42 \mathrm{~cm}$ with a grand mean of $4.68 \mathrm{~cm}$. California Wonder $(22.42 \mathrm{~cm})$ recorded the highest fruit width followed by IHR $3478(15.57 \mathrm{~cm})$, whereas, Meghalaya Local $(2.09 \mathrm{~cm})$ recorded the least fruit width. Fifteen genotypes recorded significantly higher fruit diameter than grand mean. Padhar and Zaveri (2010), Arup et al., (2011), Lakshmi and Padma (2012) and Vijaya et al., (2014) also reported same trend of range for number of fruits and fruit length. 
Table.1 Germplasm accessions of chilli (Capsicum аппиит L.)

\begin{tabular}{|c|c|c|}
\hline Treatments & Accession number or Varieties & Source \\
\hline $\mathbf{T}_{1}$ & IHR 1485 & $\begin{array}{l}\text { Indian Institute of Horticultural } \\
\text { Research, Bengaluru }\end{array}$ \\
\hline $\mathbf{T}_{2}$ & IHR 1732 & IIHR, Bengaluru \\
\hline $\mathbf{T}_{3}$ & IHR 2452 & IIHR, Bengaluru \\
\hline $\mathbf{T}_{4}$ & IHR 2596 & IIHR, Bengaluru \\
\hline $\mathbf{T}_{5}$ & IHR 2900 & IIHR, Bengaluru \\
\hline$T_{6}$ & IHR 3014 & IIHR, Bengaluru \\
\hline $\mathbf{T}_{7}$ & IHR 3024 & IIHR, Bengaluru \\
\hline $\mathbf{T}_{8}$ & IHR 3310 & IIHR, Bengaluru \\
\hline $\mathbf{T}_{9}$ & IHR 3315 & IIHR, Bengaluru \\
\hline$T_{10}$ & IHR 3443 & IIHR, Bengaluru \\
\hline$T_{11}$ & IHR 3447 & IIHR, Bengaluru \\
\hline $\mathbf{T}_{12}$ & IHR 3448 & IIHR, Bengaluru \\
\hline$T_{13}$ & IHR 3449 & IIHR, Bengaluru \\
\hline $\mathbf{T}_{14}$ & IHR 3455 & IIHR, Bengaluru \\
\hline$T_{15}$ & IHR 3478 & IIHR, Bengaluru \\
\hline$T_{16}$ & IHR 3517 & IIHR, Bengaluru \\
\hline $\mathbf{T}_{17}$ & IHR 3587 & IIHR, Bengaluru \\
\hline$T_{18}$ & IHR 3915 & IIHR, Bengaluru \\
\hline$T_{19}$ & IHR 4597 & IIHR, Bengaluru \\
\hline $\mathbf{T}_{20}$ & IHR 4595 & IIHR, Bengaluru \\
\hline $\mathbf{T}_{21}$ & IHR 4598 & IIHR, Bengaluru \\
\hline $\mathbf{T}_{22}$ & IHR 4600 & IIHR, Bengaluru \\
\hline $\mathbf{T}_{23}$ & IHR 4601 & IIHR, Bengaluru \\
\hline $\mathbf{T}_{24}$ & IHR 4602 & IIHR, Bengaluru \\
\hline$T_{25}$ & IHR 4603 & IIHR, Bengaluru \\
\hline $\mathbf{T}_{26}$ & IHR 4604 & IIHR, Bengaluru \\
\hline $\mathbf{T}_{27}$ & IHR 4605 & IIHR, Bengaluru \\
\hline$T_{28}$ & IHR 4606 & IIHR, Bengaluru \\
\hline$T_{29}$ & IHR 4607 & IIHR, Bengaluru \\
\hline $\mathbf{T}_{30}$ & IHR 4608 & IIHR, Bengaluru \\
\hline $\mathbf{T}_{31}$ & IHR 4609 & IIHR, Bengaluru \\
\hline $\mathbf{T}_{32}$ & IHR 4610 & IIHR, Bengaluru \\
\hline $\mathbf{T}_{33}$ & IHR 4611 & IIHR, Bengaluru \\
\hline $\mathbf{T}_{34}$ & IHR 4612 & IIHR, Bengaluru \\
\hline$T_{35}$ & IHR 4031 & IIHR, Bengaluru \\
\hline $\mathbf{T}_{36}$ & IHR 4516 & IIHR, Bengaluru \\
\hline $\mathbf{T}_{37}$ & IHR 4592 & IIHR, Bengaluru \\
\hline$T_{38}$ & IHR 4593 & IIHR, Bengaluru \\
\hline
\end{tabular}




\begin{tabular}{|c|c|c|}
\hline $\mathbf{T}_{\mathbf{3 9}}$ & IHR 4594 & IIHR, Bengaluru \\
\hline $\mathbf{T}_{\mathbf{4 0}}$ & G3 & $\begin{array}{c}\text { Horticultural Research Station, } \\
\text { Lam farm, Guntur }\end{array}$ \\
\hline $\mathbf{T}_{\mathbf{4 1}}$ & G4 & HRS, Lam farm, Guntur \\
\hline $\mathbf{T}_{\mathbf{4 2}}$ & G5 & HRS, Lam farm, Guntur \\
\hline $\mathbf{T}_{\mathbf{4 3}}$ & LCA 206 & HRS, Lam farm, Guntur \\
\hline $\mathbf{T}_{\mathbf{4 4}}$ & LCA 235 & HRS, Lam farm, Guntur \\
\hline $\mathbf{T}_{\mathbf{4 5}}$ & LCA 305 & HRS, Lam farm, Guntur \\
\hline $\mathbf{T}_{\mathbf{4 6}}$ & LCA 334 & HRS, Lam farm, Guntur \\
\hline $\mathbf{T}_{\mathbf{4 7}}$ & LCA 353 & HRS, Lam farm, Guntur \\
\hline $\mathbf{T}_{\mathbf{4 8}}$ & LCA 620 & HRS, Lam farm, Guntur \\
\hline $\mathbf{T}_{\mathbf{4 9}}$ & LCA 625 & HRS, Lam farm, Guntur \\
\hline $\mathbf{T}_{\mathbf{5 0}}$ & LCA 960 & HRS, Lam farm, Guntur \\
\hline $\mathbf{T}_{\mathbf{5 1}}$ & BhutJolokia & Tura, Meghalaya \\
\hline $\mathbf{T}_{\mathbf{5 2}}$ & Meghalaya Local & Tura, Meghalaya \\
\hline $\mathbf{T}_{\mathbf{5 3}}$ & CaliforniaWonder & Namdhari Seed Company \\
\hline
\end{tabular}

Table.2 Analysis of variance for yield, yield attributing traits in chilli

\begin{tabular}{|c|c|c|c|c|}
\hline \multirow[t]{2}{*}{ S. No } & \multirow[t]{2}{*}{ Character } & \multicolumn{3}{|c|}{ Mean sum of Squares } \\
\hline & & Replications & Treatments & Error \\
\hline 1. & Plant height $(\mathrm{cm})$ & 28.54 & $601.62 * *$ & 33.62 \\
\hline 2. & Plant spread $\left(\mathrm{cm}^{2}\right)$ & 43.86 & $336.19 * *$ & 30.03 \\
\hline 3. & Number of primary branches per plant & 0.34 & $0.87 * *$ & 0.12 \\
\hline 4. & Days to $50 \%$ flowering & 11.84 & $74.03 * *$ & 6.29 \\
\hline 5. & Days to $50 \%$ ripening & 31.13 & $104.82 * *$ & 21.56 \\
\hline 6. & Number of fruits per plant & 1830.81 & $41832.40 * *$ & 1706.66 \\
\hline 7. & Fruit length $(\mathrm{cm})$ & 0.030 & $21.18 * *$ & 0.128 \\
\hline 8. & Fruit width $(\mathrm{cm})$ & 0.26 & $34.01 * *$ & 0.09 \\
\hline 9. & Fruit weight & 0.22 & $423.80 * *$ & 0.17 \\
\hline 10. & Number of seeds per fruit & 32.36 & $2023.27 * *$ & 19.28 \\
\hline 11. & Seed weight (g/1000 seed) & 0.03 & $4.19 * *$ & 0.03 \\
\hline 12. & Red ripe fruit yield (g/plant) & 10125.08 & $72208.23 * *$ & 13671.31 \\
\hline
\end{tabular}


Table.3 Mean performance of yield, yield contributing traits of various chilli genotypes

\begin{tabular}{|c|c|c|c|c|c|c|c|c|c|c|c|c|c|}
\hline $\begin{array}{c}\text { S. } \\
\text { No }\end{array}$ & Genotypes & $\begin{array}{l}\text { Plant } \\
\text { height } \\
(\mathrm{cm})\end{array}$ & $\begin{array}{c}\text { Plant } \\
\text { spread } \\
\left(\mathrm{cm}^{2}\right)\end{array}$ & $\begin{array}{c}\text { No of } \\
\text { primary } \\
\text { branches/ } \\
\text { plant }\end{array}$ & $\begin{array}{c}\text { Days to } \\
50 \% \\
\text { flowering }\end{array}$ & $\begin{array}{c}\text { Days to } \\
50 \% \\
\text { ripening }\end{array}$ & $\begin{array}{l}\text { No of } \\
\text { fruits/ } \\
\text { plant }\end{array}$ & $\begin{array}{l}\text { Fruit } \\
\text { length } \\
(\mathrm{cm})\end{array}$ & $\begin{array}{l}\text { Fruit } \\
\text { width } \\
(\mathrm{cm})\end{array}$ & $\begin{array}{l}\text { Fruit } \\
\text { weight } \\
(\mathrm{g})\end{array}$ & $\begin{array}{l}\text { Number } \\
\text { of seeds } \\
\text { per fruit }\end{array}$ & $\begin{array}{c}\text { Seed } \\
\text { weight } \\
(\mathrm{g} / 1000 \\
\text { seed })\end{array}$ & $\begin{array}{l}\text { Red ripe } \\
\text { fruit yield } \\
\text { (g/plant) }\end{array}$ \\
\hline $\mathbf{T}_{1}$ & IHR 1485 & 71.53 & 47.05 & 3.86 & 46.33 & 85.67 & 264.55 & 4.96 & 3.17 & 2.12 & 54.47 & 4.47 & 555.33 \\
\hline $\mathbf{T}_{2}$ & IHR 1732 & 61.20 & 56.06 & 3.60 & 43.00 & 83.67 & 256.00 & 8.01 & 3.49 & 2.82 & 72.07 & 4.44 & 678.89 \\
\hline$T_{3}$ & IHR 2452 & 57.13 & 55.17 & 3.60 & 36.33 & 79.33 & 212.44 & 4.84 & 3.53 & 1.84 & 69.87 & 5.62 & 355.44 \\
\hline $\mathbf{T}_{4}$ & IHR 2596 & 44.20 & 55.77 & 2.87 & 32.00 & 76.67 & 116.78 & 5.78 & 4.86 & 4.62 & 88.20 & 6.35 & 470.78 \\
\hline $\mathbf{T}_{5}$ & IHR 2900 & 42.07 & 53.43 & 4.13 & 27.33 & 68.67 & 300.00 & 7.38 & 3.45 & 2.80 & 73.47 & 4.38 & 637.55 \\
\hline$T_{6}$ & IHR 3014 & 72.73 & 58.63 & 4.33 & 39.67 & 77.33 & 256.44 & 5.08 & 2.86 & 1.39 & 56.93 & 3.83 & 345.22 \\
\hline $\mathbf{T}_{7}$ & IHR 3024 & 39.40 & 58.00 & 3.67 & 30.67 & 71.67 & 306.11 & 7.82 & 2.63 & 1.97 & 79.27 & 4.95 & 502.22 \\
\hline $\mathbf{T}_{8}$ & IHR 3310 & 50.27 & 58.87 & 3.67 & 31.33 & 73.00 & 156.56 & 10.47 & 5.07 & 5.84 & 127.47 & 6.05 & 816.78 \\
\hline$T_{9}$ & IHR 3315 & 52.27 & 60.47 & 2.53 & 29.67 & 69.00 & 72.00 & 13.00 & 5.48 & 9.53 & 124.13 & 5.42 & 655.56 \\
\hline$T_{10}$ & IHR 3443 & 64.53 & 63.33 & 3.60 & 36.00 & 82.67 & 229.11 & 8.85 & 3.26 & 2.89 & 65.07 & 5.56 & 553.45 \\
\hline$T_{11}$ & IHR 3447 & 41.13 & 54.43 & 3.53 & 32.33 & 72.67 & 214.33 & 8.58 & 3.54 & 2.86 & 66.20 & 5.66 & 528.89 \\
\hline$T_{12}$ & IHR 3448 & 65.33 & 65.70 & 3.80 & 34.00 & 81.67 & 238.00 & 7.68 & 3.14 & 2.25 & 72.20 & 6.46 & 488.89 \\
\hline$T_{13}$ & IHR 3449 & 43.73 & 54.50 & 2.20 & 38.33 & 82.67 & 72.78 & 10.27 & 4.57 & 5.69 & 73.73 & 7.70 & 467.55 \\
\hline$T_{14}$ & IHR 3455 & 65.40 & 54.23 & 2.73 & 35.00 & 86.00 & 69.22 & 10.57 & 7.39 & 9.59 & 115.60 & 7.01 & 645.78 \\
\hline$T_{15}$ & IHR 3478 & 65.00 & 55.40 & 3.27 & 37.67 & 77.67 & 16.56 & 9.23 & 15.57 & 50.07 & 153.62 & 5.64 & 852.11 \\
\hline$T_{16}$ & IHR 3517 & 76.27 & 62.87 & 3.87 & 34.67 & 79.00 & 201.11 & 8.59 & 3.37 & 3.36 & 57.27 & 5.99 & 674.00 \\
\hline$T_{17}$ & IHR 3587 & 85.07 & 61.03 & 3.20 & 44.33 & 82.33 & 92.56 & 9.26 & 4.19 & 2.79 & 67.87 & 6.46 & 238.89 \\
\hline$T_{18}$ & IHR 3915 & 49.40 & 65.43 & 4.00 & 34.00 & 84.00 & 234.11 & 9.05 & 3.26 & 2.83 & 73.20 & 6.80 & 623.89 \\
\hline$T_{19}$ & IHR 4597 & 88.40 & 70.10 & 4.33 & 37.33 & 83.67 & 384.11 & 5.08 & 2.89 & 1.28 & 67.33 & 6.03 & 452.78 \\
\hline$T_{20}$ & IHR 4595 & 93.80 & 74.77 & 4.53 & 48.33 & 89.67 & 202.33 & 6.03 & 4.94 & 3.88 & 100.60 & 7.09 & 722.22 \\
\hline$T_{21}$ & IHR 4598 & 63.87 & 70.13 & 3.33 & 35.67 & 79.67 & 248.44 & 6.89 & 3.91 & 2.88 & 86.05 & 4.62 & 619.78 \\
\hline$T_{22}$ & IHR 4600 & 67.27 & 50.37 & 3.27 & 39.33 & 84.33 & 53.22 & 11.79 & 6.69 & 10.06 & 126.35 & 6.92 & 501.45 \\
\hline$T_{23}$ & IHR 4601 & 69.53 & 50.53 & 3.60 & 38.67 & 73.33 & 20.00 & 7.53 & 12.00 & 27.73 & 80.39 & 6.88 & 570.56 \\
\hline $\mathbf{T}_{24}$ & IHR 4602 & 77.07 & 52.17 & 4.27 & 43.33 & 80.67 & 253.00 & 6.88 & 3.01 & 2.00 & 55.33 & 5.81 & 478.56 \\
\hline $\mathbf{T}_{25}$ & IHR 4603 & 73.93 & 78.10 & 3.80 & 35.33 & 78.00 & 175.89 & 13.58 & 2.86 & 3.29 & 59.83 & 6.69 & 586.00 \\
\hline $\mathbf{T}_{26}$ & IHR 4604 & 64.07 & 49.73 & 2.73 & 34.67 & 75.00 & 51.11 & 11.63 & 7.05 & 10.04 & 92.80 & 10.07 & 510.33 \\
\hline$T_{27}$ & IHR 4605 & 43.33 & 52.47 & 2.73 & 33.33 & 83.33 & 183.33 & 3.87 & 4.01 & 2.85 & 92.00 & 5.46 & 446.22 \\
\hline$T_{28}$ & IHR 4606 & 77.53 & 60.63 & 3.27 & 37.00 & 87.67 & 85.89 & 6.38 & 3.43 & 3.22 & 75.53 & 6.17 & 259.22 \\
\hline
\end{tabular}




\begin{tabular}{|c|c|c|c|c|c|c|c|c|c|c|c|c|c|}
\hline$T_{29}$ & IHR 4607 & 45.07 & 68.43 & 3.20 & 35.67 & 80.00 & 218.33 & 5.84 & 2.44 & 2.23 & 75.67 & 5.90 & 421.22 \\
\hline $\mathbf{T}_{30}$ & IHR 4608 & 71.00 & 69.87 & 3.33 & 41.33 & 88.00 & 255.22 & 6.14 & 3.08 & 2.81 & 71.53 & 6.53 & 549.33 \\
\hline $\mathbf{T}_{31}$ & IHR 4609 & 62.40 & 67.13 & 3.47 & 34.33 & 81.33 & 127.89 & 10.90 & 4.21 & 3.35 & 100.84 & 6.53 & 373.56 \\
\hline$T_{32}$ & IHR 4610 & 68.93 & 65.37 & 3.27 & 38.67 & 79.00 & 102.11 & 10.97 & 5.58 & 7.83 & 82.53 & 7.83 & 795.33 \\
\hline $\mathbf{T}_{33}$ & IHR 4611 & 68.33 & 66.00 & 3.60 & 31.00 & 73.33 & 143.11 & 14.24 & 4.87 & 7.97 & 136.60 & 8.15 & 1028.45 \\
\hline $\mathbf{T}_{34}$ & IHR 4612 & 69.33 & 75.97 & 3.67 & 34.00 & 75.33 & 231.22 & 12.14 & 3.23 & 3.66 & 80.87 & 4.96 & 769.33 \\
\hline $\mathbf{T}_{35}$ & IHR 4031 & 64.27 & 56.50 & 3.20 & 40.67 & 82.33 & 197.66 & 8.14 & 3.17 & 3.27 & 55.67 & 6.14 & 600.67 \\
\hline $\mathbf{T}_{36}$ & IHR 4516 & 56.13 & 57.13 & 3.20 & 34.33 & 80.00 & 227.78 & 8.26 & 3.16 & 2.80 & 61.73 & 4.65 & 561.00 \\
\hline $\mathbf{T}_{37}$ & IHR 4592 & 67.47 & 62.40 & 3.47 & 36.33 & 77.67 & 280.45 & 5.95 & 4.15 & 2.18 & 50.07 & 4.37 & 540.56 \\
\hline $\mathbf{T}_{38}$ & IHR 4593 & 65.13 & 55.33 & 3.67 & 36.67 & 81.33 & 267.00 & 7.23 & 3.35 & 3.03 & 57.97 & 6.64 & 708.22 \\
\hline$T_{39}$ & IHR 4594 & 73.67 & 70.23 & 3.47 & 32.00 & 74.33 & 275.22 & 6.82 & 3.25 & 2.94 & 75.20 & 5.54 & 740.45 \\
\hline$T_{40}$ & G3 & 83.87 & 77.73 & 3.53 & 41.33 & 80.67 & 199.33 & 7.57 & 3.49 & 3.04 & 69.49 & 6.65 & 568.22 \\
\hline$T_{41}$ & G4 & 69.67 & 67.00 & 3.00 & 41.00 & 86.67 & 205.11 & 8.54 & 3.91 & 2.81 & 53.53 & 6.10 & 516.67 \\
\hline$T_{42}$ & G5 & 73.27 & 58.77 & 3.40 & 40.00 & 83.33 & 156.00 & 5.07 & 5.56 & 4.10 & 68.60 & 7.28 & 612.78 \\
\hline $\mathbf{T}_{43}$ & LCA 206 & 78.47 & 67.03 & 3.20 & 40.67 & 86.00 & 193.44 & 7.29 & 3.82 & 3.69 & 52.40 & 7.06 & 643.44 \\
\hline$T_{44}$ & LCA 235 & 51.13 & 58.27 & 2.87 & 36.00 & 79.00 & 257.33 & 5.16 & 3.05 & 1.78 & 79.10 & 5.97 & 376.45 \\
\hline$T_{45}$ & LCA 305 & 56.87 & 65.93 & 3.27 & 37.67 & 80.67 & 217.89 & 7.40 & 3.76 & 2.68 & 69.20 & 6.73 & 513.78 \\
\hline$T_{46}$ & LCA 334 & 62.47 & 61.87 & 3.13 & 41.00 & 87.67 & 218.78 & 6.94 & 3.45 & 2.53 & 65.20 & 5.85 & 515.11 \\
\hline$T_{47}$ & LCA 353 & 63.47 & 65.10 & 3.07 & 39.00 & 85.33 & 242.22 & 8.23 & 2.98 & 2.28 & 58.73 & 4.93 & 508.11 \\
\hline $\mathbf{T}_{48}$ & LCA 620 & 66.47 & 65.80 & 3.27 & 41.33 & 88.00 & 190.56 & 9.06 & 3.75 & 4.08 & 91.33 & 6.19 & 693.56 \\
\hline$T_{49}$ & LCA 625 & 61.53 & 63.37 & 3.00 & 39.00 & 87.33 & 232.11 & 8.13 & 3.08 & 2.42 & 59.87 & 6.11 & 549.22 \\
\hline $\mathbf{T}_{50}$ & LCA 960 & 84.40 & 60.07 & 2.67 & 38.67 & 81.33 & 129.00 & 9.21 & 5.23 & 5.59 & 115.93 & 7.38 & 770.00 \\
\hline $\mathbf{T}_{51}$ & BhutJolokia & 81.27 & 92.07 & 3.13 & 52.67 & 95.33 & 102.00 & 5.30 & 7.50 & 3.33 & 28.27 & 4.40 & 298.00 \\
\hline $\mathbf{T}_{52}$ & $\begin{array}{l}\text { Meghalaya } \\
\text { Local }\end{array}$ & 109.33 & 108.07 & 5.33 & 49.33 & 97.67 & 798.67 & 2.15 & 2.09 & 0.52 & 24.40 & 3.38 & 410.45 \\
\hline \multirow[t]{7}{*}{$\mathbf{T}_{53}$} & $\begin{array}{l}\text { California } \\
\text { Wonder }\end{array}$ & 57.13 & 52.83 & 3.60 & 36.67 & 76.00 & 11.44 & 14.48 & 22.42 & 72.47 & 118.27 & 6.63 & 759.00 \\
\hline & Mean & 65.60 & 62.79 & 3.44 & 37.64 & 81.07 & 197.09 & 8.12 & 4.68 & 6.34 & 77.92 & 6.05 & 567.19 \\
\hline & SEm \pm & 3.35 & 3.16 & 0.21 & 1.45 & 2.68 & 23.85 & 0.21 & 0.18 & 0.24 & 2.54 & 0.11 & 67.51 \\
\hline & C.D at $\overline{5} \%$ & 9.39 & 8.87 & 0.58 & 4.06 & 7.52 & 66.89 & 0.58 & 0.51 & 0.67 & 7.11 & 0.30 & 189.32 \\
\hline & C.V & 8.84 & 8.73 & 10.47 & 6.67 & 5.73 & 20.96 & 4.42 & 6.68 & 6.58 & 5.64 & 3.08 & 20.61 \\
\hline & Lowest & 39.40 & 47.05 & 2.20 & 27.33 & 68.67 & 11.44 & 2.15 & 2.09 & 0.52 & 24.40 & 3.38 & 238.89 \\
\hline & Highest & 109.33 & 108.07 & 5.33 & 52.67 & 97.67 & 798.67 & 14.48 & 22.42 & 72.47 & 153.62 & 10.07 & 1028.45 \\
\hline
\end{tabular}


Fruit weight ranged from $0.52 \mathrm{~g}$ to $72.47 \mathrm{~g}$ with a grand mean of $6.34 \mathrm{~g}$. Highest fruit weight was recorded in California Wonder(72.47 g) which was on par with IHR $3478(50.07 \mathrm{~g})$, whereas, the lowest fruit weight was recorded in Meghalaya Local(0.52 g). Nine genotypes recorded significantly more fruit weight than overall mean. These findings were in accordance with earlier reports of Gupta et al., (2009). Number of seeds per fruit varied from 24.40 to 153.62 with an average of 77.92 IHR 3478 (153.62) recorded the highest number of seeds per fruit and the lowest number of seeds per fruit was observed in Meghalaya Local (24.40). Twenty genotypes recorded more number of seeds per fruit than overall grand mean. Seed weight ranged from 3.38 to $10.07 \mathrm{~g}$ with an average of $6.05 \mathrm{~g}$. Highest seed weight was recorded in IHR 4604 (10.07g) followed by IHR 4611 $(8.15 \mathrm{~g})$, whereas, the lowest seed weight was recorded in Meghalaya Local(3.38g).Twenty eight genotypes recorded significantly more seed weight compared to overall mean. Similar range was reported by Shirshat et al., (2007) and Arup et al., (2011).

Range of red ripe fruit yield per plant varied from $238.89 \mathrm{~g}$ to $1028.45 \mathrm{~g}$ with a mean of $567.19 \mathrm{~g}$. Highest red ripe fruit yield was recorded in IHR 4611 (1028.45g) which is on par with IHR 3478 (852.11g), while IHR 3587 $(238.89 \mathrm{~g})$ recorded lowest red ripe fruit yield per plant.

Twenty five genotypes recorded significantly more red ripe fruit yield compared to overall grand mean. Suryakumari et al., (2010) and Kumar et al., (2012) were also observed wider range of variation between the genotypes studied. The characters showing wide range of variation provide an ample scope for selecting superior types and the selected genotypes can be used in further crossing programme for introgression of their desired genes and to obtain heterotic hybrids.
It is concluded in the present study as, a high range of variability was observed for all the characters. It was maximum for red ripe fruit yield per plant (238.89 to $1028.45 \mathrm{~g}$ ), followed by number of fruits per plant (11.44 to 798.67), number of seeds per fruit (24.40 to 153.62) and minimum for number of primary branches per plant $(2.20$ to $5.33 \mathrm{~cm})$ and 1000 seed weight $(0.30$ to $3.38 \mathrm{~g})$. The characters showing wide range of variation provide an ample scope for selecting superior types and the selected genotypes can be used in breeding programme for introgression of their desired genes into the high yielding varieties. The genotypes which perform better for various yield traits may be further evaluate to find the best one at other location to use in breeding programme.

\section{Acknowledgements}

I extend my gratitude to the Dr. Y. S. R. Horticultural University, Venkataramanna gudem for providing financial support in the form of stipend to complete this endeavor. I am highly thankful to ICAR - Indian Institute of Horticultural Research (IIHR), Bengaluru, Karnataka for providing research material.

\section{References}

Arup, C., B. S. Amit, N. Dai and S. Dutta. 2011. Diversity of genetic resources and genetic association analyses of green and dry chillies of Eastern India. Chilean. J. Agric. Res., 71: 3.

Bharadwaj, D. N., H. Singh and R. K. Yadav. 2007. Genetic variability and association of component characters for yield in chilli (Capsicum annuum L.). Prog. Agric., 7(1/2): 72-74.

Bhattacharya, A., A. Chattopadhyay, D. Mazumdar, A. Chakravarty and S. Pal (2010). Antioxidant constituents and enzyme activities in chilli peppers. Intl. J. Veg. Sci., 16(3): 201-211.

Bosland, P. W. and E. J. Votava. 2000. Peppers: Vegetable and spice capsicums. CABI 
Publishing, CAB International, Walingfort, U.K.

Das, B., M. S. Hazarica and P. K. Das. 1998. Studies on variability and correlation studies in fruit characters of tomato (Lycopersicon esculentum Mill.). Ann. Agri. Res., 19(1): 77-80.

FAOSTAT, Food and Agriculture Organization Corporate Statistical Database. 2016. http://faostat.fao.org/ site/339/default.aspx

Farhad, M., M. Hasanuzzaman, B. K. Biswas, A. K. Azad and M. Arifuzzaman. 2008. Reliability of yield contributing characters for improving yield potential in chilli (Capsicum annum L.). Intl. J. Sustain. Crop Prod., 3: 30-38.

Gupta, A. M., D. Singh and A. Kumar. 2009. Genetic variability, genetic advance and correlation in chilli (Capsicum annuum L.). Indian. J. Agr. Sci., 79: 221-223.

Kogure, K., S. Goto, M. Nishimura, M. Yasumoto, K. Abe and L. Ohiwa. 2002. Mechanism of potent antiperoxidative effect of capsaicin. Biochimicaet Biophysica Acta, 1573: 84-92.

Kumar, D., V. Bahadur, S. B. Rangare and D. Singh. 2012. Genetic variability, heritability and correlation studies in chilli (Capsicum annuum L.). Hort. Flora Res. Spectrum, 1: 248-252.

Lakshmi R, and Padma V. 2012. Studies on performance, genetic variability, heritability and genetic advances in chilli (Capsicum annuиm L.) varieties in high altitude and tribal zone of Srikakulam district of Andhra pradesh, India. Plant Archives, 12(2): 717720.

Munshi, A. D., B. K. Kumar, A. K. Sureja and S. Joshi. 2010. Genetic variability, heritability and genetic advance for growth, yield and quality traits in chilli. Indian J. Hort., 67(1): 114-116.

National Horticulture Board (NHB). 2017. Data

\section{How to cite this article:}

Mopidevi M. Nagaraju, R.V.S.K. Reddy, K. Madhavi Reddy, L. Naram Naidu, A. Snehalata Rani and Uma Krishna, K. 2018. Performance of Chilli (Capsicum annuum L.) Genotypes for Yield and Yield Attributing Traits. Int.J.Curr.Microbiol.App.Sci. 7(12): 2252-2260.

doi: https://doi.org/10.20546/ijcmas.2018.712.256
Base of Horticulture crops, Gurgaon, New Delhi.

Nehru, S. D., M. N. Thimmegowda and M. Gowda. 2012. Growth, yield, genetic variability and correlation studies in chilli (Capsicum annum L.). Res. J. Agric. Sci., 3(2): 517-519.

Padhar, P. R. and P. P. Zaveri. 2010. Genetic studies in relation to selection criteria in chilli. Res. on Crops, 11(3): 722-727.

Panse, V.G and Sukhatme, P.V. 1985. Statistical methods for agricultural workers, Indian Council of Agricultural Research, New Delhi.

Peter, K. V. and B. Rai (1978). Heterosis as a function of genetic distance in tomato. Ind. J. Genet. Plant Breeding, 38(2): 173-178.

Shirshat, S. S., V. A. Giritammannavar and S. J. Patil (2007). Analysis of genetic variability for quantitative traits in chilli. Karnataka $J$. Agric. Sci., 20(1): 29 -32. Singh, Y., M. Sharma and A.

Sumathy, K. M. A. and A. G. Mathew 1984. Chilli processing. Indian Cocoa, Arecanut and Spice J., 7: 112-113.

Suryakumari, S., K. Umajyothi, D. Srihari, A. S. Sankar and C. R. Sankar. 2010. Variability and genetic divergence in paprika (Capsicum annuum L.). J. Spi. Arom. Crops, 19: 71-75.

Tembhurne, B. V., R. Revanappa and P. H. Kuchanur. 2008. Varietal performance, genetic variability and correlation studies in chilli (Capsicum annuum L.). Karnataka J. Agric. Sci., 21(4): 541-543.

Vijaya, H. M., A. P. M. Gowda, S. D. Nehru and K. Jyothi. 2014. Performance of chilli (Capsicum annuum L.) genotypes for growth and yield parameters in eastern dry zone of Karnataka. J. Spi. Arom. Crops, 23(2): 250-253. 